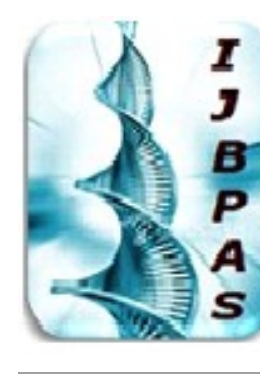

International Journal of Biology, Pharmacy and Allied Seiences (IJBPAS)

'A Bridgesetueen Caboratory and QRendor'

WwW.ijbpas.com

\title{
AN UNUSUAL PRESENTATION OF FEMORAL HERNIA
}

\section{RAJASEKARAN $\mathbf{S}^{1^{*}}$, PRABHU $\mathbf{R}^{2}$ AND VENKATESAN $\mathrm{K}^{2}$}

1: Professor and Director, Sri Lakshmi Narayana Institute of Medical Sciences (SLIMS), Pondicherry, India

2: Assistant Professor, Department of General Surgery, Sri Lakshmi Narayana Institute of Medical Sciences (SLIMS), Pondicherry, India

*Corresponding Author: Dr. S. Rajasekaran: E Mail: s.raseran@gmail.com

Received $19^{\text {th }}$ Oct. 2020; Revised $17^{\text {th }}$ Nov. 2020; Accepted 14 ${ }^{\text {th }}$ Dec. 2020; Available online $1^{\text {st }}$ Jan. 2021

https://doi.org/10.31032/IJBPAS/2021/10.1.1019

ABSTRACT

Femoral hernias $(\mathrm{FH})$ are relatively uncommon, however they are the most common incarcerated abdominal hernia with strangulation of a omentum, preperitonial fat, small bowel or colon carrying significant mortality. We report a rare case of a 72 years old female presented with symptoms of irreducible FH containing omentum. Initially, this case was misdiagnosed as an inguinal hernia outside so the ultrasound diagnosis of FH is reviewed. Lockwood surgery was performed with meshplasty. The post operative period was unremarkable and the patient was discharged as a day care surgery. This case is highlighted for the importance of examining saphenous opening in all abdominal examinations and early management of FH to prevent further complication.

\section{Keywords: Femoral hernia, Lockwood surgery, Groin swelling}

\section{INTRODUCTION}

FH account for around $2-8 \%$ of abdominal hernias and four to five times more commonly in females than males due to the wider anatomy of the female pelvis and have a peak incidence in those between 30 and 60 years old. FH can be difficult to diagnose and are at high risk of strangulation [1]. 


\section{Case Study}

A 72 years old female presented with pain in left groin for 4 months duration which was sudden in onset, progressive in nature and radiating to back. She did not have fever, nausea, anorexia, or any obstructive symptoms. No other significant past and personal history. The other systemic examinations were normal. Abdominal examination revealed a tender lump in the right groin measuring $2 \times 2 \mathrm{~cm}$ in diameter that was irreducible (Figure 1). However, a cough impulse could not be demonstrated, and the overlying skin was not erythematous. Rectal examination was normal. Patient is further evaluated with blood investigations which are within normal limits \& USG abdomen and pelvis shows features of $\mathrm{FH}$ with omentum as content. A Lockwood infra inguinal approach was made and sac identified, separated from surrounding structures and content found to be omentum (Figure 2), sac opened and content pushed inside through the saphenous ring (Figure 3) and strengthening was done. Mesh repair done in the normal way (Figure 4, 5). Wound closed in layers and skin was closed with staplers (Figure 6). Post-operative period was uneventful and the patient was discharged as a day care surgery.

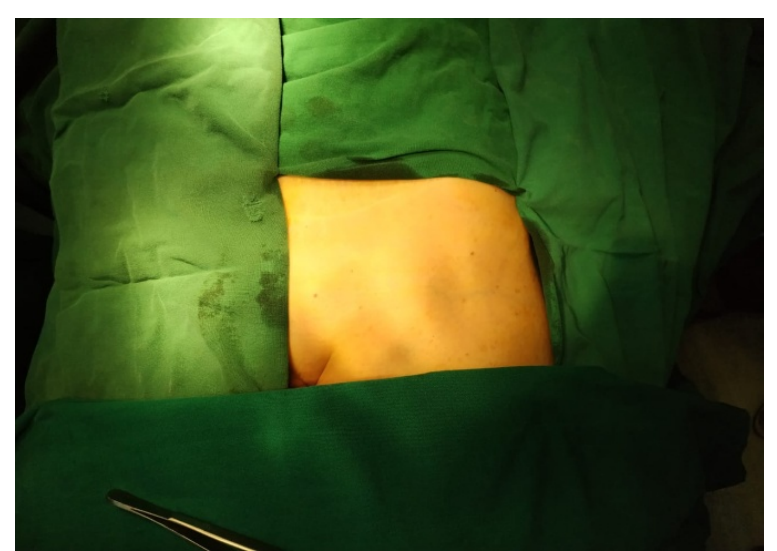

Figure 1

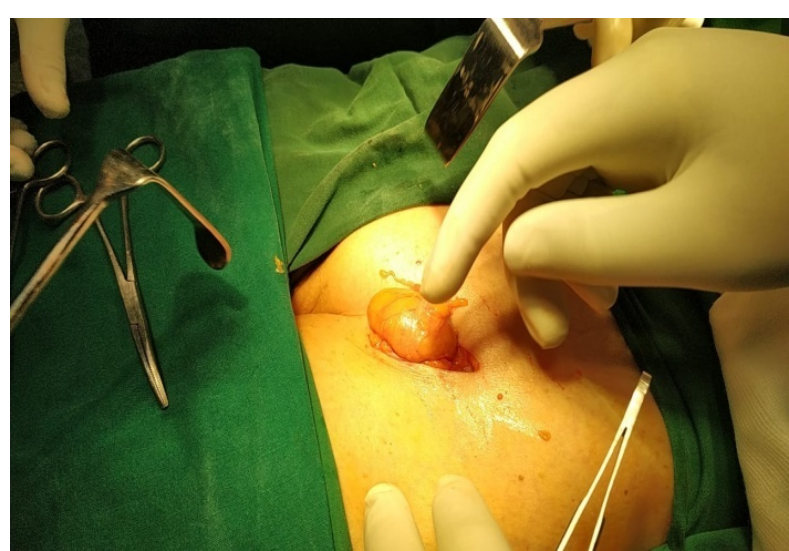

Figure 2 


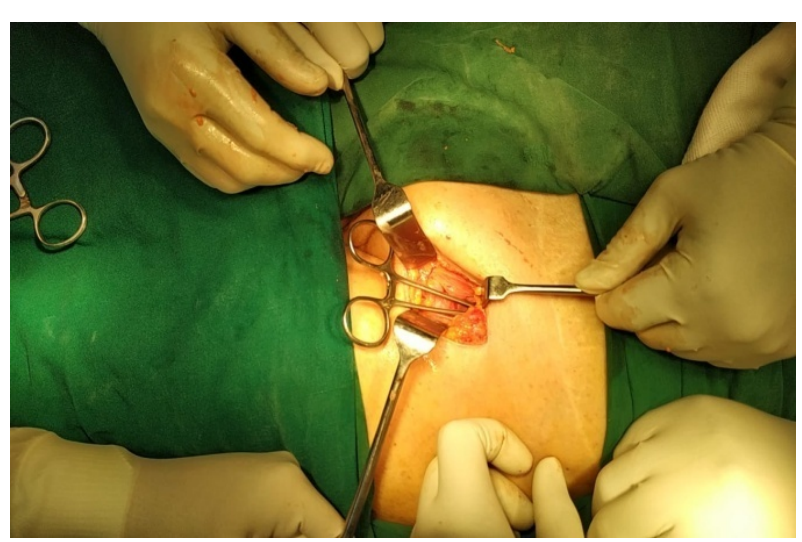

Figure 3

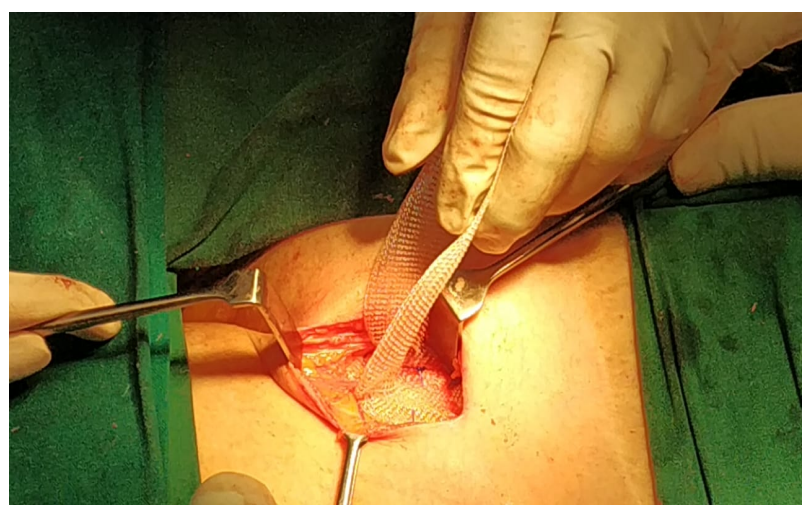

Figure 5

\section{DISCUSSION}

The FH can be usually diagnosed clinical. Most patients present as an emergency with symptoms and signs of intestinal obstruction. The femoral canal is a compartment of the femoral sheath which is bordered anterosuperiorly by the inguinal ligament, medially by the lacuna ligament, laterally by the femoral vein, and posteriorly by the pectineal ligament. Due to the small potential space in the femoral canal, femoral hernias are much more likely to become incarcerated and strangulated.

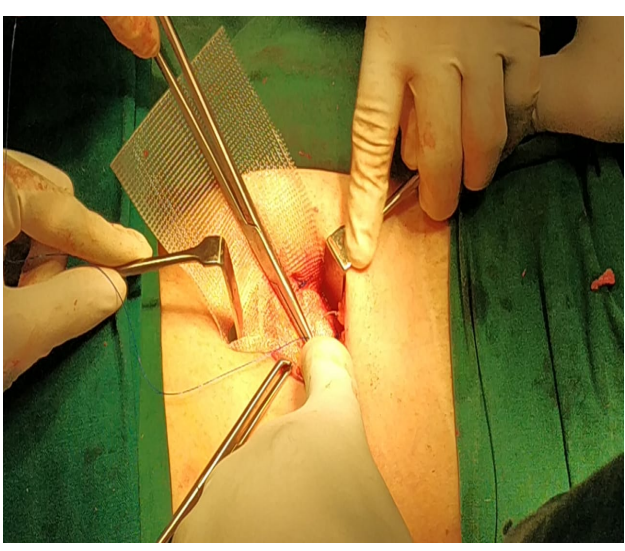

Figure 4

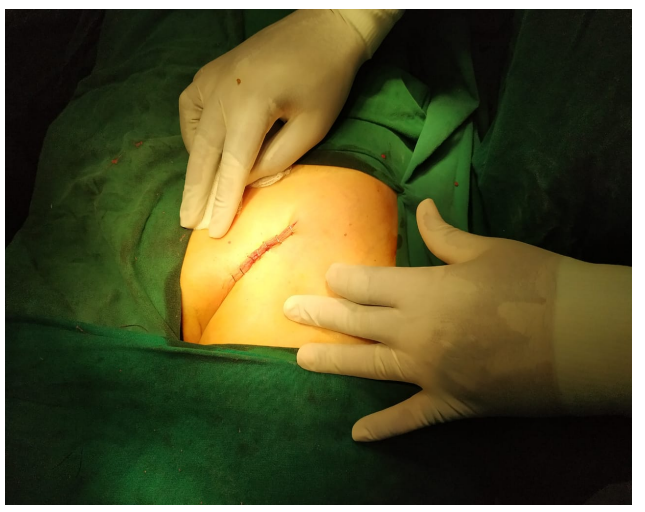

Figure 6

Femoral hernia have the highest rate of incarceration amongst groin hernias constituting about 5\%-20\% [1], because of the narrow and rigid femoral canal and therefore require early surgical repair. However, a typical FH presents as a tender, nonreducible swelling with no cough impulse and is situated below and lateral to the pubic tubercle. On exploration, it often contains only omentum, or may contain a knuckle of bowel known as Richter's hernia. The differential diagnoses include inguinal hernia, lipoma, saphena varix, enlarged lymph nodes, femoral artery aneurysm, 
sarcoma, obturator hernia, psoas abscess, psoas bursa, and in males, ectopic testis [2].

The preoperative diagnosis of femoral hernia is a challenging issue. In previous reports, the clinical diagnostic accuracy ranged from $25 \%$ to $40 \%$ [4]. In the case of de Garengeot's hernia, this can result in appendicitis and eventually perforation and abscess formation. Due to the vestigial nature of the appendix, strangulation does not result in mechanical obstruction, although ileus can occasionally develop secondary to inflammation. The tight neck of the femoral canal acts as a seal which limits inflammation and infection in the hernia sac; therefore perforation of the appendix in the hernia sac will rarely present with peritonitis. It has even been reported that, after the spontaneous reduction of a perforated appendix, the hernia neck seals off the infected collection, preventing peritoneal involvement [5].

Most case reports recommend that hernial defects should be repaired with nonabsorbable sutures. The main reason is said to be to reduce the risk of wound site infection, quoted in one study as high as $23 \%$ [6]. It is thought that the introduction of foreign material into a potentially contaminated surgical field could further increase the risk of infection $[7,8]$. The risk versus benefit of mesh repair must be considered especially in cases with large hernia defects to reduce the risk of hernia recurrence. However, the use of mesh for repair of the hernia defect is contraindicated if it is starangulated or perforated. Recent studies have supported prosthetic mesh repair, as there is less recurrence of the hernia and no increased rate of wound infection [3].

Hence in all patients with abdominal pain a thorough examination of the hernial orifices including saphenous opening examination must be carried out. Even if a tender lump is not found in the groin, the diagnosis of femoral hernia should be considered. early management is preferred to prevent morbidity and mortality of the patients.

\section{REFERENCES}

[1] Strangulated femoral hernia. Thomas D Med J Aust. 1967 Feb 11; 1(6): 258-61.

[2] A. Shah and H. S. Janardhan, "De Garengeot hernia: a case report and review of literature," Indian Journal of Surgery, vol. 75 , no. 1, pp. 439441, 2013.

[3] Sharma H, Jha PK, Shekhawat NS, Memon B, Memon MA. De Garengeot hernia: an analysis of our 
experience. Hernia. 2007; 11(3): 235 -

238.

[4] Al Shanafey S, Giacomantonio M. Femoral hernia in children. J Pediatr Surg 1999; 34: 11041106.

[5] T.-F. Hsiao and Y.-H. Chou, "Appendiceal pus in a hernia sac simulating strangulated femoral hernia: a case report," International Journal of General Medicine, vol. 4, pp. 235-237, 2011.

[6] E. T. Nguyen and I. K. Komenaka, "Strangulated femoral hernia containing a perforated appendix," Canadian Journal of Surgery, vol. 47, no. 1, pp. 68-69, 2004.

[7] H. Sharma, P. K. Jha, N. S. Shekhawat, B. Memon, and M. A. Memon, "De Garengeot hernia: an analysis of our experience," Hernia, vol. 11, no. 3, pp. 235-238, 2007.

[8] T. Piperos, V. Kalles, Y. Al Ahwal, E. Konstantinou, G. Skarpas, and T. Mariolis- Sapsakos, "Clinical significance of de Garengeot's hernia: a case of acute appendicitis and review of the literature," International Journal of Surgery Case Reports, vol. 3, no. 3, pp. 116-117, 2012. 